\title{
Comparison of two forms of strain decomposition in an elastic-plastic damaging model for concrete
}

\author{
L Contrafatto and M Cuomo \\ Department of Civil and Environmental Engineering University of Catania, vle A. Doria, 6 , \\ 95125 Catania, haly
}

\begin{abstract}
In the paper a new modified form of the constitutive equations for concrete in the general framework for elastic-plastic damaging models proposed by the authors in (Contrafatto and Cuomo 2006 J. Plast. 22 2273-300) is presented. The modification concerns the definition of the internal energy potential. In the original paper the expression of the elastic energy potential depends on the sign of the trace of the elastic strain tensor. In the new formulation a decomposition of the strain tensor in its positive and negative component by means of a basisfree representation in terms of eigenprojections is used. As a consequence a different evolution of damage, affecting in a different way the tensile and compressive component of the strain tensor, is obtained. The two models belong to the class of continuum scalar damage models and are developed within the context of simple materials. The new model, first formulated in an arbitrary cartesian coordinate system, is presented in a principal axes representation, in order to reduce the algebraic complexity of the expressions and to make easier the analysis of simple load processes, while the treatment of the constitutive equations for the general case will be an object of future developments. A comparison between the predictions of the two models is performed by means of the analysis of some loading processes. The new formulation is able to overcome some drawbacks of the original model, especially in the tensile regime. In contrast, in the compressive regime, for which already the original formulation yielded satisfactory results, no change was detected.
\end{abstract}

(Some figures in this article are in colour only in the electronic version) 


\section{Introduction}

A wide variety of theoretical models for concrete are available in the literature. They can be divided into two principal classes: models in the context of fracture mechanics and models in the context of continuum damage mechanics. The paper addresses the latter case in which damage is represented by phenomenological internal variables. The advantages and the drawbacks of a continuum model have been exhaustively treated in the literature and are not discussed here. It is well established that damage in brittle materials is inherently a nonisotropic phenomenon and a number of anisotropic damage models for concrete have also been proposed introducing 4 th or more frequently 2 nd order tensors. Since experimental evidence on concrete shows different crisis mechanisms according to the stress state (Van Mier 1984), usually limit criteria in terms of all three invariants of the stress tensor are used, like Willam or Ottosen criteria or others (Chen 1982), eventually coupled with a limit cap for tensile stresses and/or for volumetric compression. In addition a damage activation law must be provided. Anisotropic models still present two order of problems: firstly, the number of the material parameters is higher than in standard scalar models and secondly, it is usually difficult to identify all of them directly from experiments; furthermore, in the computational implementation dramatic convergence problems are often met. For these reasons many authors still use and propose isotropic damage formulations, based on a single scalar damage variable (Brenchic and Gambarotta 2001, Salari et al 2004) or, alternatively, two damage scalar variables, in an attempt to distinguish between tension and compression damage mechanisms (Comi and Perego 2001, Marfia et al 2004, Contrafatto and Cuomo 2006). Isotropic damage models have been shown to be sufficiently accurate in a wide range of practical applications, provided that the load path does not excessively rotate during the simulation.

In a previous work the authors presented a framework of elastic-plastic damaging model for concrete under multiaxial stress states (Contrafatto and Cuomo 2006), where an extensive-although not exhaustive-close examination of the concerning literature is reported. Distinctive features of that proposal are the introduction of fully coupled damage and plasticity multiple crisis criteria, ruling simultaneously the activation of all the irreversible phenomena, i.e. hardening, damage and fracture-like deformations. Therefore the limit strength is predicted by the model and turns out to be dependent on the load history; the different behaviour of concrete in tension and compression is modelled by means of a generalization of Curnier's bimodular materials (Curnier et al 1993), considering an extended kinematic space that contains, in addition to the elastic strains, the damage internal variables. In the previous paper the strain space is divided into a compression domain and a tension domain and the separating interface is defined by the deviatoric plane, as in the original Curnier proposal, so that the elastic energy takes different forms according to the sign of the trace of the elastic strain tensor. This choice is commonly assumed in isotropic damage models (Comi and Perego 2001, Faria et al 1998). It was shown that this decomposition yields very reasonable results in many simple cases. However, some limitations were also underlined, about the prediction of the lateral strain in confined compression tests and the simulation of cyclic tests, where the change in the slope of the unloading stress-strain curve not necessarily occurs at zero stress, since it is ruled by the sign of the trace of the elastic strain tensor. It is finally recalled that in nonisotropic damage models the separating interfaces are generally defined as the eigenvalues of the elastic strain tensor, often splitting it into its tensile and compressive components by means of a polar decomposition (Papa and Taliercio 1996). The same decomposition is adopted by Lubarda et al (1994).

The dependence of the elastic energy, and then of the elastic stiffness, on the variation of sign of a principal strain appears intuitively to have a sound physical basis. Although 
this decomposition is also possible in conjunction with isotropic models, in the literature it cannot be found a comparison with the decomposition based on the trace of the strain tensor. The purpose of the present paper is to perform such a comparison, implementing the new decomposition based on the sign of the principal strains in the general framework presented in (Contrafatto and Cuomo 2006), in order to verify whether it can overcome some of the drawbacks previously highlighted. With this aim a new definition of the elastic energy is introduced, employing the decomposition of the elastic strain tensor in its positive and negative components performed by means of a spectral representation. However, classical spectral decompositions cannot be safely used, since in typical load condition such as uniaxial or hydrostatic states the eigenvectors are not univocally defined, because the eigenvalues presents, respectively, double or triple coalescence and the usual split of the strain tensor cannot be performed. This difficulty can be overcome by making use of the so-called basis-free representation based on a spectral decomposition in term of eigenprojections (Carlson and Hoger 1986). This representation of the strain tensor has the advantage that it avoids a numerically expensive determination of eigenvectors and is univocally defined also in the case of defective eigenvalues. The derivation of the elastic constitutive equations and of the elastic tangent moduli is rather complex since it requires the determination of the first and second derivative of the positive and negative component of the strain tensor, i.e. it is necessary to calculate the derivative of a tensor-valued tensor function with respect to its argument. The mathematical treatment of this topic is beyond the purposes of this paper and will not be presented. Since the goal of the work is to perform a comparison of the two formulations for simple loading processes, only the relevant expressions in a principal axes representation will be reported, in the case that the classical spectral decomposition can be used, i.e. for distinct eigenvalues. In this way formulas become much simpler and can be more easily analysed.

After the introduction of the field variables in section 2, in section 3 the functional ruling the reversible behaviour is described and the derivation of the generalized elastic constitutive equations is presented. In section 4 the limit criteria are reported. Section 5 contains comparisons of the present model with the one based on the trace of the elastic strain tensor and some applications.

\section{Field variables and definition of the problem}

We refer to the model presented in (Contrafatto and Cuomo 2006). It is a continuum damage model that falls within the category of simple material models (Germain 1973). The variables of the model are

$$
\begin{array}{llll}
\varepsilon \in \mathcal{D} & \text { macroscopic strain } & \sigma \in \mathcal{D} & \text { stress } \\
\alpha \in R & \text { isotropic hardening } & \chi \in R & \text { hardening internal forces } \\
\alpha^{v} \in R & \text { volumetric hardening } & \chi^{v} \in R & \text { volumetric hardening internal forces } \\
\omega_{1} \in R & \text { tensile damage } & \zeta_{1} \in R & \text { tensile damage driving forces } \\
\omega_{2} \in R & \text { compressive damage } & \zeta_{2} \in R & \text { compressive damage driving forces }
\end{array}
$$

$\mathcal{D}$ being the space of the 2 nd order symmetric tensors.

Note that the internal variables are all scalar functions, since kinematic hardening is not considered important for concrete. As mentioned in the introduction the damage variables are considered as scalars: this is an obvious limitation, but it allows computational simplifications. Two different scalar damage variables in principle associated with tensile and compressive strain processes are used.

The model is implemented in the hypothesis of small deformations and each kinematic variable is additively decomposed in a reversible and an irreversible component, in a classic 


$$
\varepsilon=\varepsilon_{e}+\varepsilon_{p} \quad\left\{\begin{array} { c } 
{ \alpha = \alpha _ { e } + \alpha _ { p } = 0 } \\
{ \alpha ^ { v } = \alpha _ { e } ^ { v } + \alpha _ { p } ^ { v } = 0 }
\end{array} \quad \left\{\begin{array}{l}
\omega_{1}=\omega_{1_{e}}+\omega_{1_{p}}=0 \\
\omega_{2}=\omega_{2_{e}}+\omega_{2_{p}}=0
\end{array}\right.\right.
$$

Note that the definition of traditional damage models of the damage variables $\omega_{i_{p}}=$ $-\omega_{i_{e}} \in[1,0]$ has been employed.

With compact notation the state of the system is described by the two sets of dual kinematic and static state variables $\eta$ and $\tau$ :

$$
\eta=\left(\varepsilon, \alpha, \alpha^{v}, \omega_{1}, \omega_{2}\right)=\eta_{e}+\eta_{p} \quad \tau=\left(\sigma, \chi, \chi^{v}, \zeta_{1}, \zeta_{2}\right) .
$$

The evolution of the state of the material is ruled by two functionals, the internal energy $e\left(\eta_{e}\right)$ and the rate of dissipation $\mathrm{d}\left(\dot{\eta}_{p}\right)$. The apparently redundant distinction between reversible and irreversible components of the internal damage variables (i.e. $\omega_{e}=-\omega_{p}$ ) is mainly motivated by the intention of distinguishing the kinematic variables that are responsible for storage energy and for dissipation, as stated in (Contrafatto and Cuomo 2002).

By standard thermodynamic arguments the driving forces $\tau$, dual to the kinematic variables $\eta_{e}$, are obtained differentiating the free energy

$$
\tau \in \partial_{\eta_{e}} e\left(\eta_{e}\right),
$$

where the symbol $\partial$ denotes sub-differentiation, in order to account for non-smooth energy functionals.

The model closely follows the framework of paper (Contrafatto and Cuomo 2006) and the reader is cross-referred to the cited paper for details and physical motivations. However the main ingredients and the essential equations for the comprehension of the development of the present model will be recalled in sections 3 and 4 .

\section{Internal stored energy potential}

The stored energy, function of the reversible components of all the kinematic variables, is given by the sum of the elastic deformation energy $\phi\left(\varepsilon_{e}, \omega_{1_{e}}, \omega_{2_{e}}\right)$ and of the hardening (configuration) energy $\psi_{1}\left(\alpha_{e}, \omega_{1_{e}}, \omega_{2_{e}}\right)+\psi_{2}\left(\alpha_{e}^{v}\right)$, both coupled with damage:

$$
\begin{gathered}
e\left(\varepsilon_{e}, \alpha_{e}, \alpha_{e}^{v}, \omega_{1_{e}}, \omega_{2_{e}}\right)=\begin{array}{r}
\quad \\
\quad+\left(\varepsilon_{e}, \omega_{1_{e}}, \omega_{2_{e}}\right)+\psi_{1}\left(\alpha_{e}, \omega_{1_{e}}, \omega_{2_{e}}\right)+\psi_{2}\left(\alpha_{e}^{v}\right) \\
\quad \text { ind } W_{1}\left(\omega_{1_{e}}\right)+\text { ind } W_{2}\left(\omega_{2_{e}}\right),
\end{array} \\
W_{1}=\left\{\omega_{1_{e}}:-1 \leqslant \omega_{1_{e}} \leqslant 0\right\} \quad W_{2}=\left\{\omega_{2_{e}}:-1 \leqslant \omega_{2_{e}} \leqslant 0\right\} \\
\text { ind } W\left(\omega_{e}\right)=\left\{\begin{array}{cll}
0 & \text { if } & \omega_{e} \in W, \\
+\infty & \text { if } & \omega_{e} \notin W,
\end{array}\right.
\end{gathered}
$$

where the last two terms enforce the admissibility constraints for the damage variables.

\subsection{Hardening potentials}

The hardening potential $\psi_{1}\left(\alpha_{e}, \omega_{1_{e}}, \omega_{2_{e}}\right)$ that rules the evolution of the elastic domain is taken in the same form as in (Contrafatto and Cuomo 2006)

$$
\begin{aligned}
\psi_{1}\left(\alpha_{e}, \omega_{1_{e}}, \omega_{2_{e}}\right)=\frac{1}{2} & H f\left(\omega_{1_{e}}, \omega_{2_{e}}\right) \alpha_{e}^{2}, \\
& f\left(\omega_{1_{e}}, \omega_{2_{e}}\right)=p_{1}\left(1+\omega_{1_{e}}\right)^{n_{1}}+p_{2}\left(1+\omega_{2_{e}}\right)^{n_{2}}, \\
& p_{1}+p_{2}=1 \quad p_{1}, p_{2} \in R .
\end{aligned}
$$

In (3) $H$ is the hardening modulus, $p_{1}$ and $p_{2}$ are weight scalars setting the influence degree of tensile and compressive damage on hardening. Thanks to the structure of formula (3) it is possible to model the non linear ascending branch of the stress-strain behaviour of concrete, 
without introducing ad hoc rules. Moreover, the onset of damage, observed well before the peak stress is reached, is correctly reproduced.

The volumetric hardening potential $\psi_{2}\left(\alpha_{e}^{v}\right)$ accounts for the recovery of stiffness following initial degradation when concrete is loaded along the hydrostatic compression axis. According to experimental observations it has been defined as (Resende 1987)

$$
\psi_{2}\left(\alpha_{e}^{v}\right)=D \frac{a-\alpha_{e}^{v}}{a}\left[\ln \frac{a-\alpha_{e}^{v}}{a}-1\right],
$$

where $a$ is the maximum degree of compaction and $D$ has the dimension of an elastic stiffness. The dimensionless ratio $\frac{a-\alpha_{e}^{v}}{a}$ ranges between 0 and 1 .

\subsection{Elastic energy potential}

The elastic energy potential $\phi\left(\varepsilon_{e}, \omega_{1_{e}}, \omega_{2_{e}}\right)$ is function of the elastic strains and of both the damage variables, so that the elastic moduli evolve differently for tensile or compressive processes. This result can be achieved following a general methodology for bimodular elastic materials introduced by Curnier et al (1993). It consists in subdividing the strain space into a compression domain $\mathcal{D}^{-}$and a tension domain $\mathcal{D}^{+}$, separated by an hypersurface (separating interface) $z\left(\varepsilon_{e}\right)$ and in assigning a different form of the elastic energy in each sub-domain:

$$
\phi=\left\{\begin{array}{l}
\phi^{+}\left(\varepsilon_{e}, \omega_{1_{e}}, \omega_{2_{e}}\right)=\frac{1}{2}\left[2 G^{+} \varepsilon_{e}: \varepsilon_{e}+\lambda^{+} t r^{2} \varepsilon_{e}\right] \quad \text { if } \quad z\left(\varepsilon_{e}\right) \geqslant 0, \\
\phi^{-}\left(\varepsilon_{e}, \omega_{1_{e}}, \omega_{2_{e}}\right)=\frac{1}{2}\left[2 G^{-} \varepsilon_{e}: \varepsilon_{e}+\lambda^{-} t r^{2} \varepsilon_{e}\right] \quad \text { if } \quad z\left(\varepsilon_{e}\right) \leqslant 0,
\end{array}\right.
$$

where $G^{+}, G^{-}, \lambda^{+}, \lambda^{-}$are the generalized Lame coefficients, all depending on the pair $\left(\omega_{1_{e}}, \omega_{2_{e}}\right)$. In (5) the internal product : between 2 nd-order tensors introduces a double contraction of the indices.

The values of the elastic moduli have to comply with some physical conditions indicated by Curnier:

- the separating interface $z\left(\varepsilon_{e}\right)$ must contain the origin of the strain space;

- the positive and negative subdomains $\mathcal{D}^{+}$and $\mathcal{D}^{-}$must be simply connected;

- the elastic energy must be continuous across the interface

$$
\phi\left(\varepsilon_{e}, \omega_{1_{e}}, \omega_{2_{e}}\right)=\phi^{+}\left(\varepsilon_{e}, \omega_{1_{e}}, \omega_{2_{e}}\right)=\phi^{-}\left(\varepsilon_{e}, \omega_{1_{e}}, \omega_{2_{e}}\right) \quad \forall \varepsilon_{e}: z\left(\varepsilon_{e}\right)=0
$$

- the gradient of the elastic energy must be continuous across the interface (continuity of the stress-strain law)

$$
\left[\phi^{+}\right],_{\varepsilon_{e}}=\left[\phi^{-}\right], \varepsilon_{e} \Rightarrow \sigma^{+}\left(\varepsilon_{e}, \omega_{1_{e}}, \omega_{2_{e}}\right)=\sigma^{-}\left(\varepsilon_{e}, \omega_{1_{e}}, \omega_{2_{e}}\right) \quad \forall \varepsilon_{e}: z\left(\varepsilon_{e}\right)=0
$$

- the jump in the hessian of the elastic energy (elastic tensor) across the interface must be normal to the interface (i.e. there is no tangential discontinuity)

$$
\left[\sigma^{+}\right],_{\varepsilon_{e}}-\left[\sigma^{-}\right],_{\varepsilon_{e}}=s\left(\eta_{e}\right)\left[z\left(\varepsilon_{e}\right)\right],_{\varepsilon_{e}} \otimes\left[z\left(\varepsilon_{e}\right)\right]_{\varepsilon_{e}} \quad \forall \varepsilon_{e}: z\left(\varepsilon_{e}\right)=0
$$

in which $s\left(\eta_{e}\right)$ is a scalar function of the elastic kinematic variables.

In (Contrafatto and Cuomo 2006) the trace of the elastic strain tensor was used as separating interface. This approach has also been followed by Comi and Perego (2001), Faria et al (1998). In such a way the elastic energy takes different forms according to the sign of the trace of the elastic strain tensor. After imposing the continuity and the jump conditions it was found that the shear modulus is constant across the interface $\operatorname{tr} \varepsilon_{e}=0$, i.e. $G^{+}\left(\omega_{1_{e}}, \omega_{2_{e}}\right)=G^{-}\left(\omega_{1_{e}}, \omega_{2_{e}}\right)$, i.e. it evolves in the same way for tensile and compressive 
processes; consequently only three parameters could be assigned independently. The following choice for the elastic moduli was made

$$
\begin{aligned}
& G^{+}\left(\omega_{1_{e}}, \omega_{2_{e}}\right)=G^{-}\left(\omega_{1_{e}}, \omega_{2_{e}}\right)=G\left(\omega_{1_{e}}, \omega_{2_{e}}\right)=G_{0}\left(1+\omega_{1_{e}}\right)^{n_{1}}\left(1+\omega_{2_{e}}\right)^{n_{2}}, \\
& \lambda^{+}\left(\omega_{1_{e}}, \omega_{2_{e}}\right)=\left[K_{0}-\frac{2}{3} G_{0}\left(1+\omega_{2_{e}}\right)^{n_{2}}\right]\left(1+\omega_{1_{e}}\right)^{n_{1}}, \\
& \lambda^{-}\left(\omega_{1_{e}}, \omega_{2_{e}}\right)=K_{0}-\frac{2}{3} G_{0}\left(1+\omega_{1_{e}}\right)^{n_{1}}\left(1+\omega_{2_{e}}\right)^{n_{2}} .
\end{aligned}
$$

$G_{0}$ and $K_{0}$ being the undamaged elastic constants.

The elastic constitutive equations were finally given by (1):

$$
\begin{aligned}
& \sigma= \begin{cases}\sigma^{+}=2 G \varepsilon_{e}+\lambda^{+} \operatorname{tr} \varepsilon_{e} & \operatorname{tr} \varepsilon_{e} \geqslant 0, \\
\sigma^{-}=2 G \varepsilon_{e}+\lambda^{-} \operatorname{tr} \varepsilon_{e} & \operatorname{tr} \varepsilon_{e} \leqslant 0,\end{cases} \\
& \chi=H f\left(\omega_{\left.1_{e}, \omega_{2_{e}}\right) \alpha_{e},}, \begin{array}{l}
D \\
\chi^{v}=\frac{D}{a} \ln \left(\frac{a}{a-\alpha_{e}^{v}}\right), \\
\zeta_{1}=\left\{\begin{array}{l}
\zeta_{1}^{+}=\frac{1}{2}\left[2 \frac{\partial G}{\partial \omega_{1_{e}}} \varepsilon_{e} \cdot \varepsilon_{e}+\frac{\partial \lambda^{+}}{\partial \omega_{1_{e}}} t^{2} \varepsilon_{e}\right]+\frac{1}{2} H \alpha_{e}^{2} \frac{\partial f}{\partial \omega_{1_{e}}}+\zeta_{1_{a}} \quad \operatorname{tr} \varepsilon_{e} \geqslant 0, \\
\zeta_{1}^{-}=\frac{1}{2}\left[2 \frac{\partial G}{\partial \omega_{1_{e}}} \varepsilon_{e} \cdot \varepsilon_{e}+\frac{\partial \lambda^{-}}{\partial \omega_{1_{e}}} t^{2} \varepsilon_{e}\right]+\frac{1}{2} H \alpha_{e}^{2} \frac{\partial f}{\partial \omega_{1_{e}}}+\zeta_{1_{a}} \quad \operatorname{tr} \varepsilon_{e} \leqslant 0,
\end{array}\right. \\
\zeta_{2}=\zeta_{2}^{+}=\zeta_{2}^{-}=\frac{1}{2}\left[2 \frac{\partial G}{\partial \omega_{2_{e}}} \varepsilon_{e} \cdot \varepsilon_{e}+\frac{\partial \lambda^{+}}{\partial \omega_{2_{e}}} t^{2} \varepsilon_{e}\right]+\frac{1}{2} H \alpha_{e}^{2} \frac{\partial f}{\partial \omega_{2_{e}}}+\zeta_{2_{a}} .
\end{array}\right.
\end{aligned}
$$

where $\zeta_{1_{a}} \in \partial \operatorname{ind} W_{1}\left(\omega_{1_{e}}\right), \zeta_{2_{a}} \in \partial \operatorname{ind} W_{2}\left(\omega_{2_{e}}\right)$

In the remaining part of this paper this model will be referred to as model MI.

An alternative choice is investigated in this work: the elastic strain tensor is split into its tensile and compressive components by means of some polar decomposition (Lubarda et al 1994, Papa and Taliercio 1996, Carlson and Hoger 1986).

Classical theories are based on the usual spectral decomposition

$$
\varepsilon_{e}=\sum_{a=1}^{3} e_{a} n_{a} \otimes n_{a} \quad\left|n_{a}\right|=1
$$

$e_{a}$ being the distinct eigenvalues, i.e. the principal strain, and $n_{a}$ the normalized eigenvectors of the elastic strain tensor $\varepsilon_{e}$, i.e. the principal direction of strain. The positive (tensile) component of the strain tensor can be obtained removing the negative eigenvalues from (8) and vice versa for the negative (compressive) component. If we introduce the Heaviside step function $h(x)$, such that

$$
h\left(e_{a}\right)=\left\{\begin{array}{ll}
1 & e_{a}>0, \\
0 & e_{a}<0,
\end{array} \quad h\left(-e_{a}\right)= \begin{cases}0 & e_{a}>0, \\
1 & e_{a}<0,\end{cases}\right.
$$

they are given by:

$$
\varepsilon_{e}^{+}=\sum_{a=1}^{3} e_{a} h\left(e_{a}\right) n_{a} \otimes n_{a} \quad \varepsilon_{e}^{-}=\sum_{a=1}^{3} e_{a} h\left(-e_{a}\right) n_{a} \otimes n_{a}
$$

and it results $\varepsilon_{e}=\varepsilon_{e}^{+}+\varepsilon_{e}^{-}$.

This decomposition is valid provided that all eigenvalues are non-defective so that their algebraic multiplicity coincides with the geometric multiplicity, i.e. with the number of associated linearly independent eigenvectors. However it presents computational difficulties related to the cases of repeated eigenvalues, typical of uniaxial traction or compression $\left(e_{2}=e_{3}\right)$ or of hydrostatic states $\left(e_{1}=e_{2}=e_{3}\right)$. In such situation the eigenvectors are not univocally defined so that the usual split of the strain tensor cannot be performed. 
When this situation occurs, the FE codes fail. It is remarkable that none of the authors that have used a similar decomposition has explicitly indicated a correct alternative procedure holding for defective eigenvalues.

For overcoming this inconvenience the so-called basis-free representation based on a spectral decomposition in term of eigenprojections is needed. This representation has the advantage that it avoids a numerically expensive determination of eigenvectors. In addition it is univocally defined also in the case of defective eigenvalues. Accordingly, the elastic strain tensor $\varepsilon_{e}$ is decomposed as it follows:

$$
\varepsilon_{e}=\varepsilon_{e}^{+}+\varepsilon_{e}^{-} \quad \varepsilon_{e}=\sum_{a=1}^{p} e_{a} M_{a},
$$

where $e_{a}$ are the $p$ distinct eigenvalues of $\varepsilon_{e}(0<p \leqslant 3)$ and $M_{a}$ are the eigenprojection univocally given by Silvester's formula:

$$
\begin{array}{lll}
M_{a}=\prod_{\substack{b \neq a \\
a=1}}^{b} \frac{\varepsilon_{e}-e_{a} I}{e_{a}-e_{b}} & p>1, \\
M_{a}=I & p=1,
\end{array} \quad \sum_{a=1}^{p} M_{a}=I, \quad M_{a} M_{b}=\left\{\begin{array}{cc}
M_{a} & a=b, \\
0 & a \neq b .
\end{array}\right.
$$

$I$ being the identity tensor.

For each component the spectral decomposition yields

$$
\varepsilon_{e}^{+}=\sum_{a=1}^{p} e_{a} h\left(e_{a}\right) M_{a} \quad \varepsilon_{e}^{-}=\sum_{a=1}^{p} e_{a} h\left(-e_{a}\right) M_{a} .
$$

The mathematical background of the decomposition in terms of eigenprojections can be found, for instance, in Carlson and Hoger (1986), Itskov and Aksel (2002), Itskov (2002) and Xiao et al (1998).

Let $G_{0}$ and $\lambda_{0}$ be the Lamè constants of a linearly elastic isotropic material and $K_{0}=\left(\frac{2}{3} G_{0}+\lambda_{0}\right)$ the bulk modulus. The standard quadratic isotropic elastic energy functional

$$
u\left(\varepsilon_{e}\right)=\frac{1}{2}\left[2 G_{0} \varepsilon_{e}: \varepsilon_{e}+\lambda_{0} t r^{2} \varepsilon_{e}\right]
$$

can be rewritten introducing the decomposition (13) of the elastic strain tensor in the form

$$
\begin{aligned}
u\left(\varepsilon_{e}\right)= & \frac{1}{2}\left[2 G_{0}\left(\varepsilon_{e}^{+}+\varepsilon_{e}^{-}\right):\left(\varepsilon_{e}^{+}+\varepsilon_{e}^{-}\right)+\lambda_{0} t^{2}\left(\varepsilon_{e}^{+}+\varepsilon_{e}^{-}\right)\right] \\
= & \frac{1}{2}\left[2 G_{0} \varepsilon_{e}^{+}: \varepsilon_{e}^{+}+2 G_{0} \varepsilon_{e}^{-}: \varepsilon_{e}^{-}+\lambda_{0}\left(\operatorname{tr}^{2} \varepsilon_{e}^{+}+\operatorname{tr}^{2} \varepsilon_{e}^{-}+2 \operatorname{tr} \varepsilon_{e}^{+} \operatorname{tr} \varepsilon_{e}^{-}\right)\right] \\
= & \frac{1}{2}\left[2 G_{0} \operatorname{dev}\left(\varepsilon_{e}^{+}\right): \operatorname{dev}\left(\varepsilon_{e}^{+}\right)+2 G_{0} \operatorname{dev}\left(\varepsilon_{e}^{-}\right): \operatorname{dev}\left(\varepsilon_{e}^{-}\right)\right] \\
& +\frac{1}{2}\left[K_{0} t r^{2} \varepsilon_{e}^{+}+K_{0} t r^{2} \varepsilon_{e}^{-}+2 \lambda_{0} \operatorname{tr} \varepsilon_{e}^{+} \operatorname{tr} \varepsilon_{e}^{-}\right],
\end{aligned}
$$

where the split in the deviator and the hydrostatic component of the strain tensor is highlighted. In the present model the elastic moduli independently evolve with damage. Therefore, the five parameters appearing in the five terms of (15) can be assumed, in principle, as independent constitutive parameters, so that the following expression for the elastic internal energy is introduced:

$$
\begin{aligned}
\phi\left(\varepsilon_{e}, \omega_{1_{e}}, \omega_{2_{e}}\right)= & \frac{1}{2}\left[2 G_{1} \operatorname{dev}\left(\varepsilon_{e}^{+}\right): \operatorname{dev}\left(\varepsilon_{e}^{+}\right)+K_{1} t r^{2} \varepsilon_{e}^{+}\right]+ \\
& \frac{1}{2}\left[2 G_{2} \operatorname{dev}\left(\varepsilon_{e}^{-}\right): \operatorname{dev}\left(\varepsilon_{e}^{-}\right)+K_{2} t^{2} \varepsilon_{e}^{-}+2 \lambda_{12} \operatorname{tr} \varepsilon_{e}^{+} t r \varepsilon_{e}^{-}\right],
\end{aligned}
$$

where $G_{1}, G_{2}, K_{1}, K_{2}, \lambda_{12}$ are generalized Lamè coefficients, all depending on the damage pair $\left(\omega_{1_{e}}, \omega_{2_{e}}\right)$.

The function (16) has to satisfy the continuity and jump conditions introduced for the bimodular material. As mentioned in the introduction, in order to reduce the algebraic complexity of the expressions, these conditions are here presented in principal axes 
representation in the case of distinct eigenvalues. The conclusions, however, possess general validity. The strain tensor components are then given by (10)

$\varepsilon_{e}^{+}=\left[\begin{array}{lll}e_{a} h\left(e_{a}\right) & & \\ & e_{b} h\left(e_{b}\right) & \\ & & e_{c} h\left(e_{c}\right)\end{array}\right] \quad \varepsilon_{e}^{-}=\left[\begin{array}{lll}e_{a} h\left(-e_{a}\right) & & \\ & e_{b} h\left(-e_{b}\right) & \\ & & e_{c} h\left(-e_{c}\right)\end{array}\right]$.

The stress tensor is obtained by the derivative of the elastic energy potential (16) w.r.t. the elastic strain tensor:

$$
\begin{aligned}
\sigma=\left[\phi\left(\varepsilon_{e}, \omega_{1_{e}}, \omega_{2_{e}}\right)\right], \varepsilon_{\varepsilon_{e}}= & {\left[\phi\left(\varepsilon_{e}, \omega_{1_{e}}, \omega_{2_{e}}\right)\right],_{\varepsilon_{e}^{+}}: H^{+}+\left[\phi\left(\varepsilon_{e}, \omega_{1_{e}}, \omega_{2_{e}}\right)\right],,_{\varepsilon_{e}^{-}}: H^{-} } \\
= & {\left[2 G_{1} \operatorname{dev}\left(\varepsilon_{e}^{+}\right)+K_{1} \operatorname{tr}\left(\varepsilon_{e}^{+}\right) I+\lambda_{12} \operatorname{tr}\left(\varepsilon_{e}^{-}\right) I\right]: H^{+} } \\
& +\left[2 G_{2} \operatorname{dev}\left(\varepsilon_{e}^{-}\right)+K_{2} \operatorname{tr}\left(\varepsilon_{e}^{-}\right) I+\lambda_{12} \operatorname{tr}\left(\varepsilon_{e}^{+}\right) I\right]: H^{-}
\end{aligned}
$$

where $H^{+}$and $H^{-}$represent the derivatives of the positive and negative component of the elastic strain w.r.t. the elastic strain tensor:

$$
H^{+}=\left[\varepsilon_{e}^{+}\right], \varepsilon_{e} \quad H^{-}=\left[\varepsilon_{e}^{-}\right], \varepsilon_{e} .
$$

It can be shown that in the principal axes representation the generic components of the stress tensor $\sigma$ and of the tangent elastic tensor $C$, in the case of distinct eigenvalues, are, respectively, given by:

$$
\begin{aligned}
\sigma_{a a}= & \left\{2 G_{1}\left[\frac{2}{3} e_{a} h\left(e_{a}\right)-\frac{1}{3} e_{b} h\left(e_{b}\right)-\frac{1}{3} e_{c} h\left(e_{c}\right)\right]+K_{1} \operatorname{tr}\left(\varepsilon_{e}^{+}\right)\right\} h\left(e_{a}\right) \\
& +\left\{2 G_{2}\left[\frac{2}{3} e_{a} h\left(-e_{a}\right)-\frac{1}{3} e_{b} h\left(-e_{b}\right)-\frac{1}{3} e_{c} h\left(-e_{c}\right)\right]+K_{2} \operatorname{tr}\left(\varepsilon_{e}^{-}\right)\right\} h\left(-e_{a}\right) \\
& +\lambda_{12}\left[\operatorname{tr}\left(\varepsilon_{e}^{+}\right) h\left(-e_{a}\right)+\operatorname{tr}\left(\varepsilon_{e}^{-}\right) h\left(e_{a}\right)\right] . \\
C_{a a b b}=\frac{\partial \sigma_{a a}}{\partial \varepsilon_{e_{b b}}} \equiv \frac{\partial \sigma_{a a}}{\partial e_{b}}= & {\left[-\frac{2}{3} G_{1} h\left(e_{b}\right)+K_{1} h\left(e_{b}\right)\right] h\left(e_{a}\right)+} \\
& +\left[-\frac{2}{3} G_{2} h\left(-e_{b}\right)+K_{2} h\left(-e_{b}\right)\right] h\left(-e_{a}\right)+\quad a \neq b \\
& +\lambda_{12}\left[h\left(e_{b}\right) h\left(-e_{a}\right)+h\left(-e_{b}\right) h\left(e_{a}\right)\right] .
\end{aligned}
$$

The continuity condition of the stress-strain law implies

$$
\lim _{e_{a} \rightarrow 0^{+}} \sigma_{a a}=\lim _{e_{a} \rightarrow 0^{-}} \sigma_{a a} .
$$

The jump condition across the interfaces implies

$$
\begin{aligned}
& \lim _{e_{b} \rightarrow 0^{+}} \frac{\partial \sigma_{a a}}{\partial e_{b}}=\lim _{e_{b} \rightarrow 0^{-}} \frac{\partial \sigma_{a a}}{\partial e_{b}} \quad a \neq b, \\
& \lim _{e_{b} \rightarrow 0^{+}} \frac{\partial \sigma_{a a}}{\partial e_{b}}-\lim _{e_{b} \rightarrow 0^{-}} \frac{\partial \sigma_{a a}}{\partial e_{b}}=s\left(\eta_{e}\right) \quad a=b
\end{aligned}
$$

From $(22 a)$ it derives that

$$
\left[-\frac{2}{3} G_{1}+K_{1}\right] h\left(e_{a}\right)+\lambda_{12} h\left(-e_{a}\right)=\left[-\frac{2}{3} G_{2}+K_{2}\right] h\left(-e_{a}\right)+\lambda_{12} h\left(e_{a}\right)
$$

so that

$$
\begin{array}{ll}
\lambda_{12}=-\frac{2}{3} G_{1}+K_{1} & \text { if } e_{a}>0 \\
\lambda_{12}=-\frac{2}{3} G_{2}+K_{2} & \text { if } e_{a}<0 .
\end{array}
$$

Using (19) condition (21) is satisfied provided that

$$
-\frac{2}{3} G_{1}+K_{1}=-\frac{2}{3} G_{2}+K_{2} \text {. }
$$


From (23) it follows that the expression of $\lambda_{12}\left(\omega_{1_{e}}, \omega_{2_{e}}\right)$ is the same for $e_{a}<0$ and $e_{a}>0$. The jump condition (22b), using (20b) and introducing equation (24), becomes

$$
2 G_{1}-2 G_{2}=s\left(\eta_{e}\right) .
$$

Conditions (23) and (24) imply that only three of the five parameters can be assigned independently and equation (25) imposes that the shear modulus must take distinct values across the interfaces.

The following choice for the elastic moduli is accordingly made

$$
\begin{aligned}
& G_{1}\left(\omega_{1_{e}}, \omega_{2_{e}}\right)=G_{0}\left(1+\omega_{1_{e}}\right)^{n_{1}}\left(1+\omega_{2_{e}}\right)^{n_{2}}, \\
& G_{2}\left(\omega_{1_{e}}, \omega_{2_{e}}\right)=G_{0}\left(1+\omega_{2_{e}}\right)^{n_{2}}, \\
& K_{1}\left(\omega_{1_{e}}, \omega_{2_{e}}\right)=K_{0}\left(1+\omega_{1_{e}}\right)^{n_{1}} .
\end{aligned}
$$

The choice is motivated by experimental evidence: in fact, the bulk modulus $K_{0}$ remains undamaged in hydrostatic compression if no previous tensile damage is present, i.e. if $\omega_{1_{e}}=0 \rightarrow K_{2}=K_{0}$. Moreover the tensile shear modulus degrades with either compressive or tensile damage, while tensile damage does not affect the shear parameter in compression $G_{2}$.

According to equations (23) and (24) the parameters $K_{2}$ and $\lambda_{12}$ take the form:

$$
\begin{aligned}
& K_{2}\left(\omega_{1_{e}}, \omega_{2_{e}}\right)=K_{0}\left(1+\omega_{1_{e}}\right)^{n_{1}}+\frac{2}{3} G_{0}\left(1+\omega_{2_{e}}\right)^{n_{2}}\left[1-\left(1+\omega_{1_{e}}\right)^{n_{1}}\right], \\
& \lambda_{12}\left(\omega_{1_{e}}, \omega_{2_{e}}\right)=\left[K_{0}-\frac{2}{3} G_{0}\left(1+\omega_{2_{e}}\right)^{n_{2}}\right]\left(1+\omega_{1_{e}}\right)^{n_{1}} .
\end{aligned}
$$

Note that, as in model MI, for undamaged processes (i.e. $\omega_{1_{e}}=\omega_{2_{e}}=0$ ), the material constants (26) and (27) coincide with the classical elastic moduli.

From definitions (16), (26), (3) and (4) the expressions of the generalized elastic energy potential and of the constitutive equations follow:

$$
\begin{aligned}
& \phi\left(\varepsilon_{e}, \omega_{1_{e}}, \omega_{2_{e}}\right)=\frac{1}{2}\left[2 G_{1} \varepsilon_{e}^{+}: \varepsilon_{e}^{+}+2 G_{2} \varepsilon_{e}^{-}: \varepsilon_{e}^{-}+\lambda_{12} t r^{2} \varepsilon_{e}\right] . \\
& \sigma=2 G_{1} \varepsilon_{e}^{+}: H^{+}+2 G_{2} \varepsilon_{e}^{-}: H^{-}+\lambda_{12} t r \varepsilon_{e} \\
& \chi=H f\left(\omega_{1_{e}}, \omega_{2_{e}}\right) \alpha_{e} \\
& \chi^{v}=\frac{D}{a} \ln \left(\frac{a}{a-\alpha_{e}^{v}}\right) \\
& \zeta_{1}=\frac{1}{2}\left[2 \frac{\partial G_{1}}{\partial \omega_{1_{e}}} \varepsilon_{e}^{+}: \varepsilon_{e}^{+}+2 \frac{\partial G_{2}}{\partial \omega_{1_{e}}} \varepsilon_{e}^{-}: \varepsilon_{e}^{-}+\frac{\partial \lambda_{12}}{\partial \omega_{1_{e}}} t r^{2} \varepsilon_{e}\right]+\frac{1}{2} H \alpha_{e}^{2} \frac{\partial f}{\partial \omega_{1_{e}}}+\zeta_{1_{a}}, \\
& \zeta_{2}=\frac{1}{2}\left[2 \frac{\partial G_{1}}{\partial \omega_{2_{e}}} \varepsilon_{e}^{+}: \varepsilon_{e}^{+}+2 \frac{\partial G_{2}}{\partial \omega_{2_{e}}} \varepsilon_{e}^{-}: \varepsilon_{e}^{-}+\frac{\partial \lambda_{12}}{\partial \omega_{2_{e}}} t r^{2} \varepsilon_{e}\right]+\frac{1}{2} H \alpha_{e}^{2} \frac{\partial f}{\partial \omega_{2_{e}}}+\zeta_{2_{a}} .
\end{aligned}
$$

where $\zeta_{1_{a}} \in \operatorname{dind} W_{1}\left(\omega_{1_{e}}\right), \zeta_{2_{a}} \in \partial \operatorname{ind} W_{2}\left(\omega_{2_{e}}\right)$

In the sequel formulas (28) and (29) will be denoted as model MII.

\section{The inelastic behaviour}

A generalized elastic domain is defined in the extended space of stresses, thermodynamic forces and conjugated damage variables, given by the convex hull of three elastic limit surfaces:

$$
\begin{array}{cl}
g(\tau)=\max \left\{g_{1}, g_{2}, g_{3}\right\} \quad & g_{1}\left(\sigma, \chi, \zeta_{2}\right)=f\left(I_{1}, J_{2}, J_{3}, \chi, \zeta_{2}\right), \\
& g_{2}\left(\sigma, \zeta_{1}\right)=\bar{f}\left(I_{1}, J_{2}\right)+\zeta_{1}-\zeta_{0}, \\
& g_{3}\left(\sigma, \chi^{v}\right)=-\sigma_{m}+\sigma_{m}^{c}-\chi^{v}, \\
I_{1}=\operatorname{tr} \sigma \quad J_{2}=\frac{1}{2} \operatorname{dev} \sigma \cdot \operatorname{dev} \sigma \quad J_{3}=\operatorname{det} \operatorname{dev} \sigma \quad \zeta_{0}, \sigma_{m}^{c} \in R \quad \sigma_{m}=\frac{1}{3} I_{1} .
\end{array}
$$


No change has been introduced in the limit criterion with respect to paper (Contrafatto and Cuomo 2006). The function $g_{1}$ is a plastic yield criterion, able to model the behaviour of concrete in the range of triaxial compression with low lateral confinement; the introduction of the isotropic hardening variable $\chi$ and of the static damage variable $\zeta_{2}$ causes the initial expansion, followed by the contraction of the domain when damage in compression is active. Specifically an extended Ottosen criterion has been adopted:

$$
\begin{aligned}
g_{1}\left(\sigma, \chi, \zeta_{2}\right) & =b_{1} I_{1}(\sigma)+\frac{b_{2}}{f_{c}^{*}} J_{2}(\sigma)+\lambda(\cos 3 \theta(\sigma)) \sqrt{J_{2}(\sigma)}-f_{c}^{*}, \\
f_{c}^{*} & =\left(f_{c}+\chi-k_{\zeta} \zeta\right) .
\end{aligned}
$$

In (30) $\theta(\sigma)$ is the Lode angle and $b_{1}, b_{2}, k_{1}$ and $k_{2}$ are material constants defining the amplitude and the shape of the deviatoric sections. The compressive strength $f_{c}^{*}$ is a function of the compression elastic limit $f_{c}$, the hardening $\chi$ and the damage $\zeta_{2}$. The parameter $k_{\zeta}$ represents an adjustable damage rate.

The hardening and damage variables evolve according to the elastic constitutive equations. The static variables $\left(\sigma, \chi, \zeta_{2}\right)$ follow a complex path in the generalized stress space; therefore the Ottosen limit state is rather different from the classical one proposed by Ottosen (1977) and it depends also on the form chosen for the internal energy, that links the evolution of the damage energy to the stress state.

The function $g_{2}$ is a damage-like criterion for tensile stresses, the limit $\zeta_{0}$ being related to the fracture energy and $\bar{f}=\alpha I_{1}(\sigma)+\beta \sqrt{J_{2}(\sigma)} \quad \alpha, \beta \in R$.

The function $g_{3}$ represents a volumetric cap along the hydrostatic axis translating with the hardening conjugated variable $\chi^{v}$.

Details about the admissible domain and the determination of the relevant parameters are given in (Contrafatto and Cuomo 2006).

\section{Comparison of the models and discussion}

\subsection{Elastic constitutive equations}

The particularization of the generalized elastic relations (7) and (29) for uniaxial and spherical stress states is reported in tables $1-3$.

A strong difference in the elastic relations between the two models is met in hydrostatic compression: in the case of model MI the process is purely elastic and the damage forces are zero while with the model MII the equivalent tangent bulk modulus undergoes a progressive reduction if $\omega_{1_{e}} \neq 0$, i.e. if some tensile damage (crack-like) had been previously suffered by the material. On the contrary, the two models present identical elastic behaviour in hydrostatic tension. Table 4 reports the values of the damage forces at the elastic limit, for some simple processes. The limit value of $\zeta_{2}$ is always the same for the two models. Recalling that for an undamaged (i.e. purely elastic) process the material parameters reduce to the elastic moduli, it follows that the same elastic limit in compression is predicted according to criterion $g_{1}$ for both models. Therefore the material parameters of Ottosen criterion can be evaluated with the same procedure described in (Contrafatto and Cuomo 2006). However, this consideration is not true for the tensile damage variable $\zeta_{1}$. Since the fracture-like criterion $g_{2}$ depends on the evolution of $\zeta_{1}$, it follows that the two models, for fixed material parameters, predict a different elastic limit in tension. Consequently the constitutive parameters in $g_{2}$ take different values for the two models.

Figure 1 presents a plot of the deformation energy of models MI and MII in plane strain for the two values of the damage variables, $\omega_{1_{e}}=-0.8$ and $\omega_{2_{e}}=-0.8$. The most remarkable difference is that for model MI the stored energy is smaller in the region $\operatorname{tr} \varepsilon_{e}>0$, while for 
Table 1. Generalized damaged static variables in uniaxial tension.

Uniaxial tension

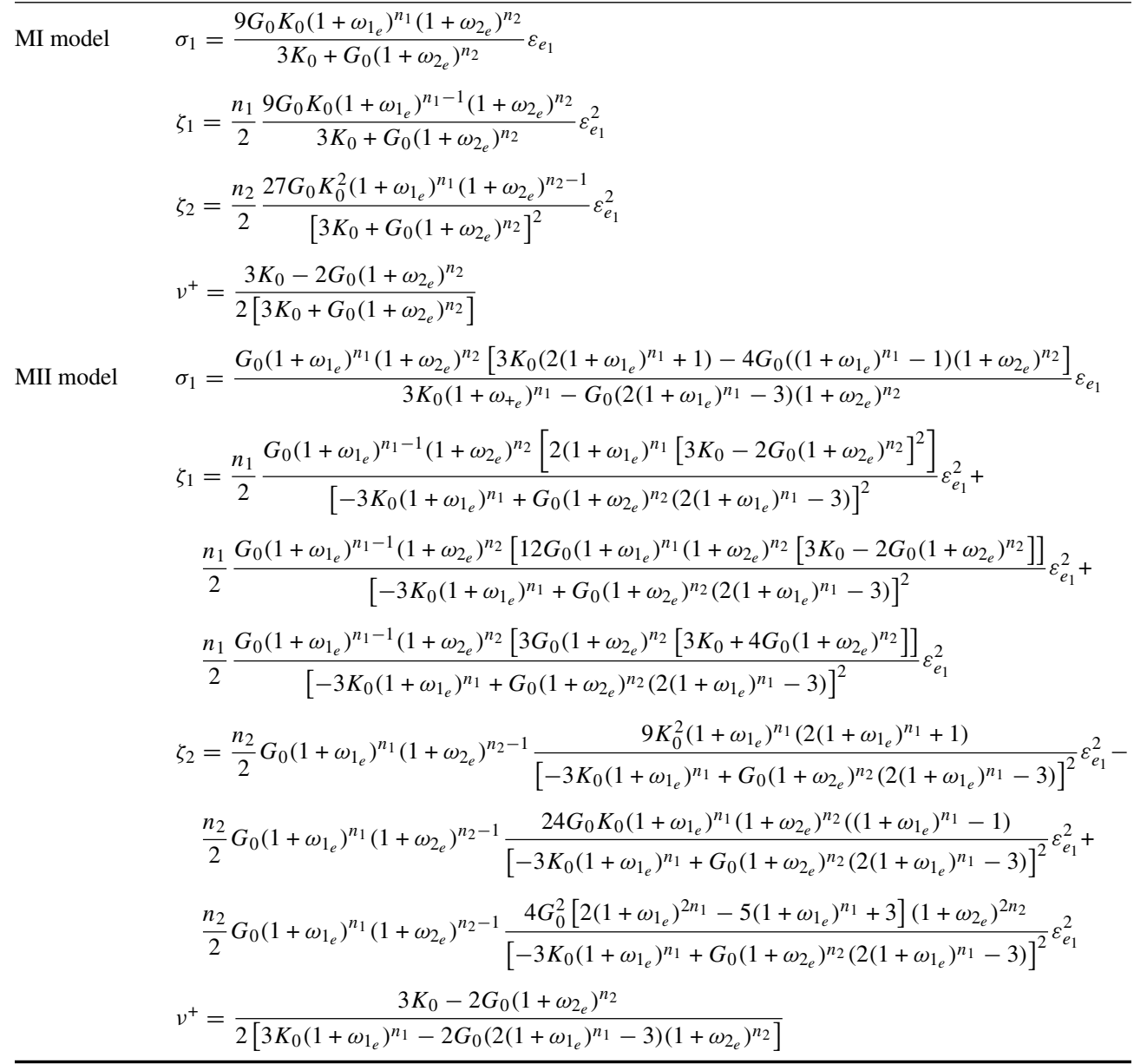

model MII the amount of the stored energy changes in each quadrant, according to the sign of the principal strains.

Figures 2 and 3 refer to uniaxial tension and compression processes, respectively.

Figures $2(a)$ and $(b)$ compare the adimensional tangent modulus in uniaxial tension as a function of tensile damage at fixed values of the compressive damage. Similar plots for the Poisson ratio are shown in figures $2(c)$ and $(d)$. While the evolution of the longitudinal stiffness is similar in the two cases, the lateral deformations evolve in a rather different fashion: model MI predicts a value of the Poisson ratio that is not affected by the tensile damage, while the same tends to decrease for model MII, according to the evolution of a fracture process, that occurs with concentrated displacement and at constant lateral strains. Thus this appears to be an improvement of the new proposal.

Also in uniaxial compression there are differences; in model MI the compression modulus is highly reduced by tensile damage. This effect is much lower in model MII. This implies that in cyclic processes Model II predicts a sharp change in the slope of the stress-strain curve upon reversal of the load. 
Table 2. Generalized damaged static variables in uniaxial compression.

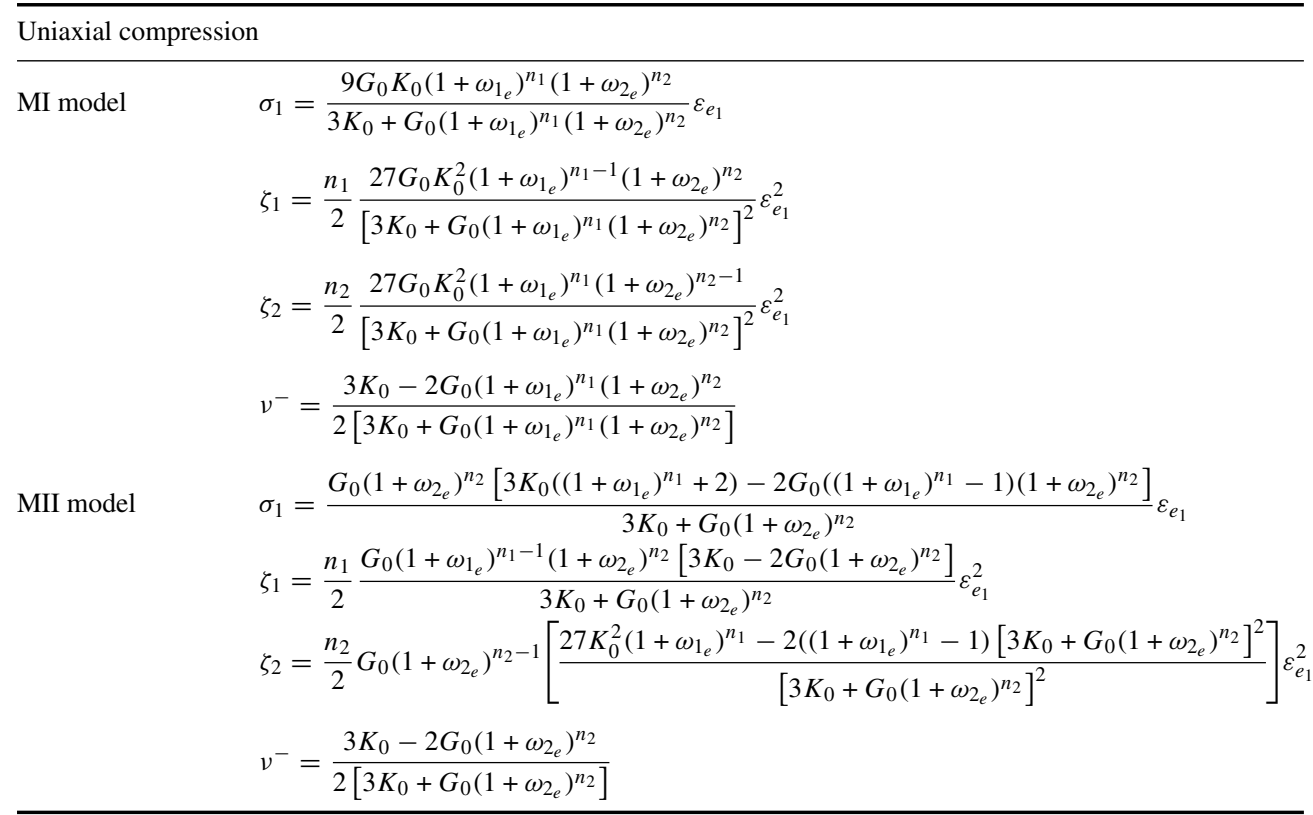

Table 3. Generalized damaged static variables in hydrostatic stress states.

Hydrostatic compression MI model

$$
\begin{aligned}
& \sigma_{m}=3 K_{0} \varepsilon_{m} \\
& \zeta_{1}=0 \\
& \zeta_{2}=0 \\
& \sigma_{m}=\left[3 K_{0}\left(1+\omega_{1_{e}}\right)^{n_{1}}-2 G_{0}\left(\left(1+\omega_{1_{e}}\right)^{n_{1}}-1\right)\left(1+\omega_{2_{e}}\right)^{n_{2}}\right] \varepsilon_{m} \\
& \zeta_{1}=\frac{n_{1}}{2}\left(1+\omega_{1_{e}}\right)^{n_{1}-1}\left[K_{0}-\frac{2}{3} G_{0}\left(1+\omega_{2_{e}}\right)^{n_{2}}\right] \varepsilon_{m}^{2} \\
& \zeta_{2}=-\frac{n_{2}}{2} \frac{2}{3} G_{0}\left(\left(1+\omega_{1_{e}}\right)^{n_{1}}-1\right)\left(1+\omega_{2_{e}}\right)^{n_{2}-1} \varepsilon_{m}^{2}
\end{aligned}
$$

MII model

Hydrostatic tension

MI model

MII model

$$
\begin{aligned}
& \sigma_{m}=3 K_{0}\left(1+\omega_{1_{e}}\right)^{n_{1}} \varepsilon_{m} \\
& \zeta_{1}=\frac{n_{1}}{2} K_{0}\left(1+\omega_{1_{e}}\right)^{n_{1}-1} \varepsilon_{m}^{2} \\
& \zeta_{2}=0 \\
& \sigma_{m}=3 K_{0}\left(1+\omega_{1_{e}}\right)^{n_{1}} \varepsilon_{m} \\
& \zeta_{1}=\frac{n_{1}}{2} K_{0}\left(1+\omega_{1_{e}}\right)^{n_{1}-1} \varepsilon_{m}^{2} \\
& \zeta_{2}=0
\end{aligned}
$$

Similarly, model MII yields a value of the Poisson ratio that remains unaffected by the tensile damage. Both models, however, predict that for very high compressive damage the deformation tends to become isochoric, and this again is compatible with the rupture that occurs for sliding of the fractures. 
Table 4. Elastic limit value of damage variables in simple load processes.

Elastic limit value of damage variables

Uniaxial tension

MI model

MII model

Uniaxial compression

MI model

MII model

Hydrostatic compression

MI model

$\zeta_{1}=n_{1} G_{0}\left(1+v_{0}\right) \varepsilon_{e_{1}}^{2}=\frac{n_{1}}{2} E_{0} \varepsilon_{e_{1}}^{2}$

$\zeta_{2}=n_{2} \frac{2}{3} G_{0}\left(1+v_{0}\right)^{2} \varepsilon_{e_{1}}^{2}=\frac{n_{2}}{3} E_{0}\left(1+v_{0}\right) \varepsilon_{e_{1}}^{2}$

$\zeta_{1}=n_{1} G_{0}\left(1-v_{0}\right)\left(1+2 v_{0}\right) \varepsilon_{e_{1}}^{2}=\frac{n_{1}}{2} E_{0} \frac{\left(1-v_{0}\right)\left(1+2 v_{0}\right)}{\left(1+v_{0}\right)} \varepsilon_{e_{1}}^{2}$

$\zeta_{2}=n_{2} \frac{2}{3} G_{0}\left(1+v_{0}\right)^{2} \varepsilon_{e_{1}}^{2}=\frac{n_{2}}{3} E_{0}\left(1+v_{0}\right) \varepsilon_{e_{1}}^{2}$

MII model

$\zeta_{1}=n_{1} \frac{2}{3} G_{0}\left(1+v_{0}\right)^{2} \varepsilon_{e_{1}}^{2}=\frac{n_{1}}{3} E_{0}\left(1+\nu_{0}\right) \varepsilon_{e_{1}}^{2}$

$\zeta_{2}=n_{2} \frac{2}{3} G_{0}\left(1+v_{0}\right)^{2} \varepsilon_{e_{1}}^{2}=\frac{n_{2}}{3} E_{0}\left(1+v_{0}\right) \varepsilon_{e_{1}}^{2}$

$\zeta_{1}=n_{1} G_{0} \nu_{0} \varepsilon_{e_{1}}^{2}=\frac{n_{1}}{2} \frac{E_{0}}{\left(1+v_{0}\right)} v_{0} \varepsilon_{e_{1}}^{2}$

$\zeta_{2}=n_{2} \frac{2}{3} G_{0}\left(1+v_{0}\right)^{2} \varepsilon_{e_{1}}^{2}=\frac{n_{2}}{3} E_{0}\left(1+v_{0}\right) \varepsilon_{e_{1}}^{2}$

Hydrostatic tension

MI model

$\zeta_{1}=0$

$\zeta_{2}=0$

$\zeta_{1}=n_{1} G_{0} \frac{\nu_{0}}{\left(1-2 v_{0}\right)} \varepsilon_{m}^{2}=\frac{n_{1}}{2} \frac{\nu_{0} E_{0}}{\left(1+\nu_{0}\right)\left(1-2 \nu_{0}\right)} \varepsilon_{m}^{2}$

$\zeta_{2}=0$

MII model

$$
\begin{aligned}
& \zeta_{1}=\frac{n_{1}}{2} K_{0} \varepsilon_{m}^{2} \\
& \zeta_{2}=0 \\
& \zeta_{1}=\frac{n_{1}}{2} K_{0} \varepsilon_{m}^{2} \\
& \zeta_{2}=0
\end{aligned}
$$

Table 5. Constitutive parameters

\begin{tabular}{ll}
\hline Initial Young's modulus & $E_{0}=22,000 \mathrm{MPa}$ \\
Initial hardening modulus & $H_{0}=20,000 \mathrm{MPa}$ \\
Initial Poisson's ratio & $\nu_{0}=0.25$ \\
Uniaxial elastic compressive strength & $f_{\mathrm{c}}=11.87 \mathrm{MPa}$ \\
Uniaxial limit tensile strength & $f_{\mathrm{t}}=1.09 \mathrm{MPa}$ \\
Equi-biaxial elastic compressive strength & $f_{\mathrm{bc}}=1.30-\mathrm{fc}=15.43 \mathrm{MPa}$ \\
Maximum principal stress in a pure shear test & $f_{\mathrm{s}}=1.10 \mathrm{MPa}$ \\
Damage parameters & $k_{\varsigma}=60-n_{2}=2$ \\
\hline
\end{tabular}

\subsection{Limit domain}

In the subsequent applications the set of material properties given in table 5 has been used, from which the following material parameters have been derived:

$$
a=1.1463 \quad b=3.5462 \quad k_{1}=12.6900 \quad k_{2}=0.9750 .
$$

The limit elastic Ottosen domain assumes the same shape in both decompositions MI and MII because the damage variable $\zeta_{2}$ has the same functional expression, as it can be observed in table 4.

The meridian sections of the elastic domain, valid for both the models MI and MII, are shown in figure 4 , for the two values of the Lode angle $\vartheta=0^{\circ}$ and $\vartheta=60^{\circ}$, corresponding to 


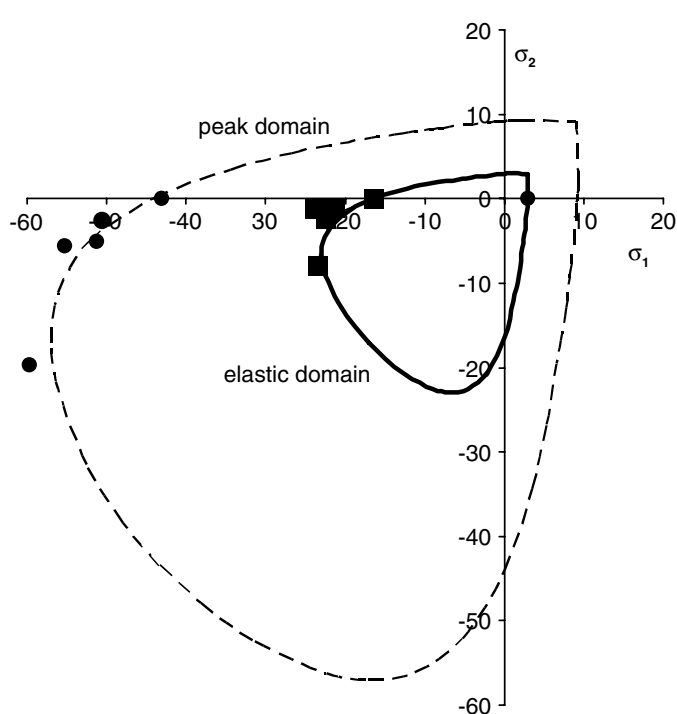

Figure 5. Comparison between Ottosen's elastic and failure limit biaxial envelope and Van Mier's experimental data.

elastic energy, the biaxial fracture domain has been plotted for three cases:

$$
\begin{aligned}
& g_{2_{0}}\left(\sigma, \zeta_{1}\right)=\zeta_{1}-\zeta_{0}, \\
& g_{2_{a}}\left(\sigma, \zeta_{1}\right)=\alpha I_{1}(\sigma)+\zeta_{1}-\zeta_{0}, \\
& g_{2_{b}}\left(\sigma, \zeta_{1}\right)=\alpha I_{1}(\sigma)+\beta \sqrt{J_{2}(\sigma)}+\zeta_{1}-\zeta_{0} .
\end{aligned}
$$

Function (31a) consists in a bound for $\zeta_{1}$, i.e. it models mode I fracture processes. The second form $(31 b)$ allows to couple the effects of damage and isotropic stress through the presence of the first invariant of the stress tensor $I_{1}$, while in the third function (31c) the dependence on the second invariant of the stress deviator $J_{2}$ includes the presence of shear across the fracture interfaces.

The predictions for the biaxial limit stresses obtained using fracture criteria $g_{2}$ with models MI and MII are compared in figure 6.

The pure damage model is almost isotropic in the tensile and compressive regions, and thus is not useful for real predictions. Criteria $g_{2_{a}}$ and $g_{2_{b}}$ show different limits for various stress ratios. Therefore models MI and MII give different predictions. The deviatoric sections of the fracture criterion are circular if model MI is used. In contrast, using model MII triangular deviatoric sections are obtained. The high compressive limit value predicted can be related to the crisis of materials that show no plastic damage due to microcracks coalescence in a compression process. This is typical of highly compact rocks, as marble or basalt that collapse with abrupt explosions, or some type of high strength concrete. The combined biaxial elastic limit domain in the range of biaxial tension is shown in figure 7 for model MII. It seems to agree better than model MI with experimental trends since it does not show an excessive reduction of the tensile strength for biaxial tensile stress states with respect to the uniaxial tensile strength.

\subsection{Uniaxial tests}

In the introduction it is observed that among the main limitations of the original model is the accurate prediction of experiments with reversed load. It is pointed out in section 5.1 that the 


\section{References}

Brenchic A and Gambarotta L 2001 Isotropic damage model with different tensile-compressive response for brittle materials Int. J. Solids Struct. 38 5865-92

Carlson D E and Hoger A 1986 The derivative of a tensor-values function of a tensor Q. Appl. Math. 44 409-23

Chen W 1982 Plasticity in Reinforced concrete (New York: McGraw-Hill)

Comi C and Perego U 2001 Fracture energy based bi-dissipative damage model for concrete Int. J. Solids Struct. 38 6427-54

Contrafatto L and Cuomo M 2002 A new thermodynamically consistent continuum model for hardening plasticity coupled with damage Int. J. Solids Struct. 39 6241-71

Contrafatto L and Cuomo M 2006 A framework of elastic-plastic damaging model for concrete under multiaxial stress states Int. J. Plast. 22 2273-300

Curnier A, He Q-C and Zysset P 1993 Conewise linear elastic materials J. Elast. 37 1-38

Faria R, Oliver J and Cervera M 1998 A strain-based plastic viscous-damage model for massive concrete structures Int. J. Solids Structures 35 1533-58

Germain P 1973 Mécanique des Milieux Continues (Masson)

Itskov M 2002 The derivative with respect to a tensor: some theoretical aspects and applications ZAMM-Z. Angew. Math. Mech. 82 535-44

Itskov M and Aksel N 2002 A closed-form representation for the derivative of non-symmetric tensor power series Int. J. Solids Struct. 39 5963-78

Lubarda V, Krajcinovic D and Mastlovic S 1994 Damage model for brittle elastic solids with unequal tensile and compressive strengthsEng. Fract. Mech. 49 681-97

Marfia S, Rinaldi Z and Sacco E 2004 Softening behavior of reiforced concrete beams under cyclic loading Int. J. Solids Struct. 41 3293-316

Ottosen N 1977 A failure criterion for concrete J. Eng. Mech. Div. (ASCE) 103 527-35

Papa E and Taliercio A 1996 Anisotropic damage model for the multiaxial static and fatigue behaviour of plain concrete Eng. Fract. Mech. 55 163-79

Reinhardt H 1984 Fracture mechanics of an elastic softening material like concrete Heron 29 1-42

Resende L 1987 A damage mechanics constitutive theory for the inelastic behaviour of concrete Comput. Methods Appl. Mech. Eng. 60 57-93

Salari M, Saeb S, Willam K, Patchet S and Carrasco R 2004 A coupled elastoplastic damage model for geomaterials Comput. Methods Appl. Mech. Eng. 193 2625-43

Taylor R 2002 FEAP - A Finite Element Analysis Program (Berkeley, CA: University of California)

Van Mier J 1984 Strain softening of concrete under multiaxial loading conditions PhD Thesis Technische Wetenschappen aan de Technische Hogeschool, Eindhoven

Xiao H, Bruhns O T and Meyers A T M 1998 Strain rates and material spins J. Elast. 52 1-41 